\title{
Study of conducting tissue in a case of ventricular pre-excitation ${ }^{1}$
}

\author{
R. H. Anderson, M. K. Thapar, R. Arnold, and R. S. Jones \\ From the Institute of Child Health, University of Liverpool, and Royal Liverpool Children's Hospital
}

A case is described which exhibited $L$ transposition with an $L$ bulboventricular loop and atrial inversion. Both great vessels arose from the left-sided right ventricle, and the electrocardiogram showed evidence of ventricular pre-excitation, with minimal abnormality of the $Q R S$ complex. Examination of the conducting tissue showed the atrioventricular node to be hypoplastic. A second node-like structure was present anteriorly in the atrial septum, and two bundles pierced the fibrous ring, passing around a sinus in the ventricular septum to form a common bundle which supplied the ventricular myocardium. These findings are discussed in relation to theories of production of the pre-excitation syndrome.

The classic form of Wolff-Parkinson-White (WPW) syndrome has been explained on the basis of lateral accessory atrioventricular connexions as described by Kent (1893). However, such connexions are not identified in all hearts exhibiting the abnormality, and these cases have been explained on the basis of the anatomical bypass tracts in the region of the atrioventricular node described by Mahaim and Benatt (1937) and James (196I). Presence of these bypass tracts also affords an explanation for the several variants of the WPW syndrome (Ferrer, 1967, 1970; Durrer, Schuilenburg, and Wellens, I970). We have recently conducted a histological examination of a heart with a WPW variant, and the results are of great interest with regard to the theories concerning pre-excitation.

\section{Case report}

A term infant weighing $2722 \mathrm{~g}$ was delivered without complications. At 4 months of age she developed a chest infection and congestive cardiac failure, and was noted to be cyanosed. She was admitted to hospital, where digitalis therapy was started, and then transferred to the Royal Liverpool Children's Hospital at 5 months of age. On admittance she weighed $3855 \mathrm{~g}$, and was found to be cyanosed and dyspnoeic, and to have dextrocardia. She had cardiomegaly and hepatomegaly, the second heart sound was single and accentuated, and a soft systolic murmur was audible at the right upper sternal edge. No diastolic murmur was audible. The electrocardiogram

${ }^{1}$ Supported by a grant from the British Heart Foundation. revealed sinus rhythm with a rate of 166 a minute. The $P$ axis was $+120^{\circ}$ and the mean $Q R S$ axis was $+75^{\circ}$. The PR interval was $0.08 \mathrm{sec}$, and the QRS complex, while not prolonged, showed initial slurring and evidence of right ventricular hypertrophy (Fig. I). $X$-ray examination confirmed dextrocardia and cardiomegaly, and showed the stomach to be right sided. Cardiac catheterization revealed arterial desaturation with a step-up at atrial level. The venous ventricular pressure was at sys-

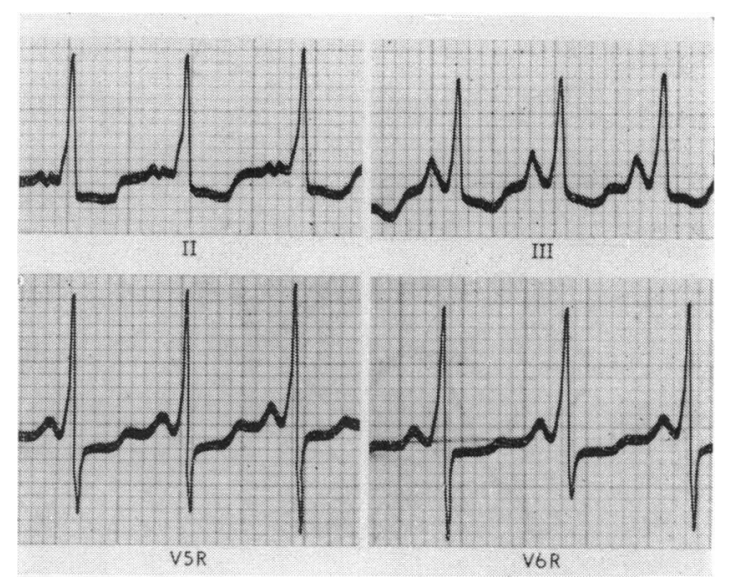

FIG. I Tracing from leads $I I, I I I, V 5 R$ and V6R. The $P R$ interval is $0.08 \mathrm{sec}$ and the $Q R S$ complex shows minimal initial slurring but its duration is not prolonged. 
temic level. Selective angiocardiography exhibited a double outlet right ventricle (left sided), and further revealed that dye was retained in the arterial atrium. After consultation it was decided that her condition was not amenable to operation, and despite intensive therapy her condition deteriorated, and she died at 7 months of age.

\section{Necropsy}

The organs revealed complete situs inversus. The heart exhibited an L-bulboventricular loop with atrial inversion. The venous atrium was left sided and received the superior and inferior venae cavae, together with a left superior vena cava. The internal morphology of the chamber was typical of the venous atrium, and it drained through a tricuspid atrioventricular valve into a leftsided ventricular chamber with the internal morphology of the normal right ventricle. Bulbar musculature separated the tricuspid orifice from the ostia of the aorta and the pulmonary artery, both of which arose from this chamber. The aortic and pulmonary valves lay side by side, with the aorta to the left. The ventricular septum was complete, but showed no evidence of a membranous portion. The right-sided ventricular chamber had the internal morphology of the normal left ventricle, but was very hypoplastic. A bicuspid atrioventricular valve separated it from the right-sided atrium, which received the four pulmonary veins and was typical of a normal left atrium. A patent foramen ovale was present.

\section{Histological examination}

A block of tissue was removed from the heart consisting of the distal interatrial and proximal interventricular septa, and enclosing both great vessels and the opening of the coronary sinus. The block was embedded in paraffin wax, sectioned at Io $\mu$ thickness, and I in 50 of the sections was mounted and stained with Masson's trichrome technique. After initial examination, I in Io of pertinent sections was mounted and stained.

The atrioventricular node was found related to the ostium of the coronary sinus beneath the endocardium of the venous atrium (left sided). However, the structure was very poorly formed. Thus attenuated transitional cells coarsed over a small collection of interweaving nodal cells, which abutted against the upward extension of the atrioventricular bundle (Fig. 2b). The atrioventricular bundle pierced the fibrous annulus, and passed posteriorly in relation to a pocket of the ventricular cavity, which passed as a blind sinus into the septum, and contained tricuspid ring and valve tissue (Fig. 2C). Beneath this sinus the bundle was joined by another fascicle of specialized tissue, which was larger than the AV bundle. This anterior bundle passed round the anterior rim of the sinus and pierced the fibrous ring, and the specialized tissue came into contact with atrial musculature forming a second atrial collection of specialized tissue (Fig. 2a). From this anterior node, extensions of specialized cells were traced anteriorly into the bases of the tricuspid (Fig. 2d) and mitral valves. Beneath the sinus the two conducting fascicles joined into a common bundle which descended directly into the musculature of the inter- ventricular septum, where it bifurcated (Fig. 2c). A cord-like bundle branch ran to the left-sided ventricular chamber, but it was not possible to trace the specialized tissue to the right-sided chamber.

\section{Discussion}

The type of pre-excitation syndrome examined approximates to the short PR, normal QRS syndrome first adequately documented by Lown, Ganong, and Levine (1952). However, these authors laid down strict criteria for their cases, one of which was heart rate below I0O, another being completely normal QRS complexes with no initial slurring. Since the present case fails to satisfy either of these criteria it should probably be described as a WPW variant, but the electrocardiogram is very similar to the case shown as Lown-Ganong-Levine syndrome in the review of Durrer et al. (I970). Be that as it may, previous reviewers have explained such cases on the basis of bypass tracts of James (Ferrer, 1967, 1970; Durrer et al., 1970) or unknown AV junction abnormalities (Durrer et al., 1970). The present case does not possess any bypass tracts such as described by James (I96I); similarly, the abnormal connexion does not approximate to the type first described by Mahaim and Benatt (1937). The latter connexion is made between the atrioventricular bundle or node and the interventricular septum, whereas in this case two well-formed bundles of specialized tissue make contact with atrial myocardium through two node-like structures. This arrangement can certainly be considered to represent previously unknown AV junction abnormalities. The posterior node related to the coronary sinus is much deficient in its upper components when compared with normal infantile AV nodes studied by one of us (Anderson and Latham, 1971). This upper portion of transitional cells and nodal fasciculi is that portion of the node usually implicated in nodal delay (Hoffman and Cranefield, 1960). The large anterior AV bundle also passes through the fibrous AV ring, and forms an anterior node-like structure in relation to the atrial musculature. However, the bulk of this structure is formed of bundle-like cells and extensions of specialized tissue pass from it into the AV rings. It is reasonable to postulate that this additional tissue is a remnant of the primitive atrioventricular canal shown to form a substantial portion of the normal AV node (Anderson and Taylor, 1972), particularly since extensions into the valve bases are seen in this case. Thus it is possible that the pre-excitation in this case may be due to either insufficient production of delay in a malformed node, or bypass of the main node by the additional atrioventricular connexion. In either case ventricular excitation would occur through the common $\mathrm{AV}$ 


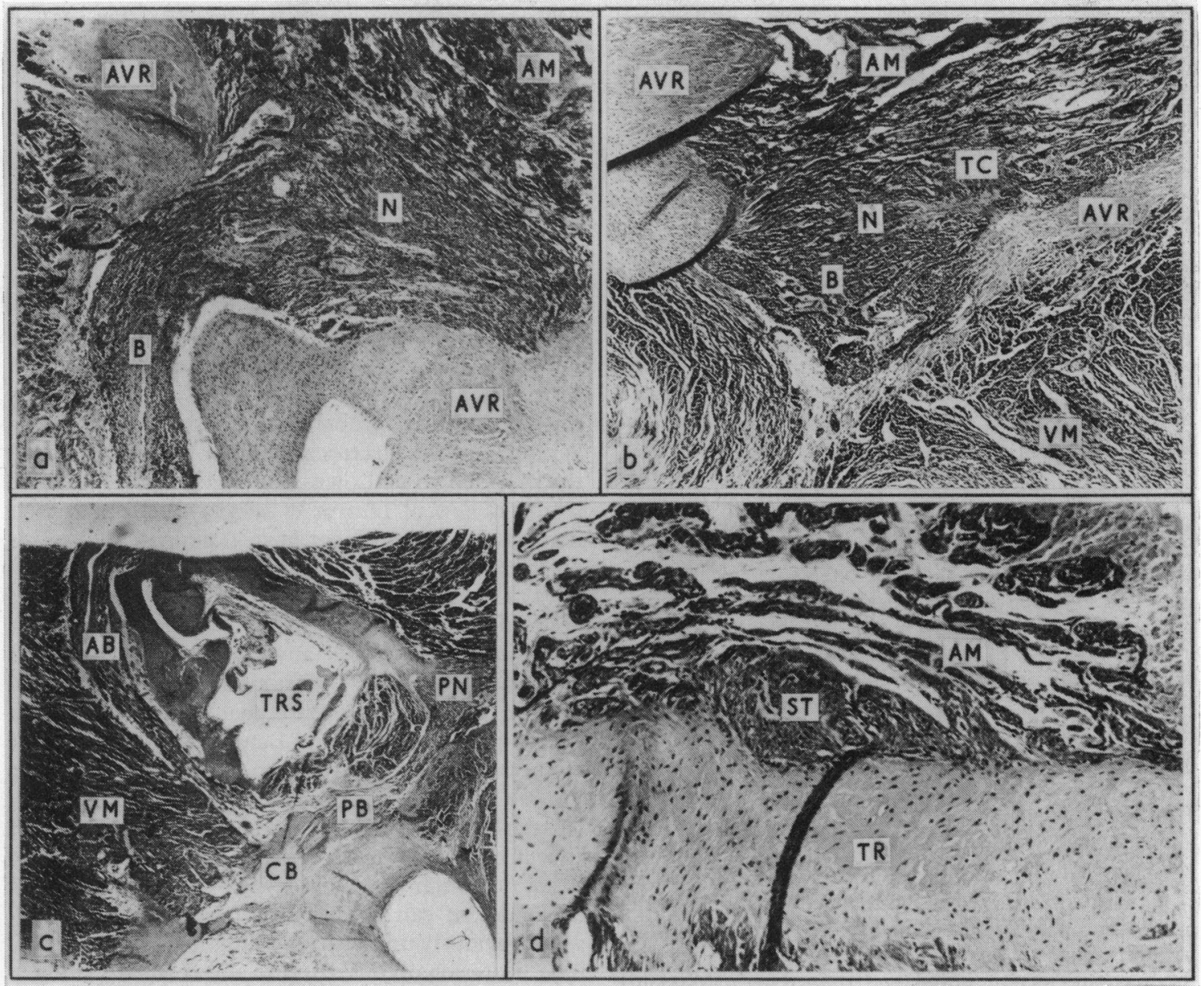

FIG. 2 Sections of the conducting tissue. (a) The anterior atrioventricular connexion showing a well-formed bundle $(B)$ piercing the fibrous atrioventricular ring $(A V R)$ to communicate with atrial myocardium $(A M)$ via a node-like structure $(N)$. (b) The normal $A V$ node is hypoplastic. The nodal element $(N)$ is small and impinges on bundle cells $(B)$ piercing the fibrous ring $A V R)$. Attenuated transitional cells (TC) are present between node and atrial myocardium $(A M) . V M$ - ventricular myocardium. (c) Lower power view showing the anterior bundle $(A B)$ and posterior bundle $(P B)$ passing around a sinus lined by tricuspid ring tissue (TRS). The communication of the posterior bundle with the node $(P N)$ is seen, and the junction of the two bundles to form a common bundle $(C B)$ which supplies the ventricular myocardium (VM). (d) The extension of the anterior node-like structure into the attachment of the atrial myocardium $(A M)$ to the tricuspid ring (TR). Note the specialized nature of the cells $(S T)$.

bundle. It may also be pertinent that at present we are examining cases of common ventricles, and several of these exhibit ventricular pre-excitation, and have a similar anterior AV bundle and poorly developed node-like structure. In these hearts, however, the posterior node makes no contact with ventricular myocardium (R.H. Anderson, M. K. Thapar, R. Arnold, and R. S. Jones, unpublished observa- tions). It is realized that the described case is an extreme example, and the heart was grossly malformed and incapable of sustaining life. It is, therefore, unjustified to draw strict comparisons between this case and the bulk of pre-excitation syndromes which occur in adults. However, it raises the possibility that the syndromes may be caused by hypoplasia of the upper part of the normal AV node rather than 
the bypassing of these nodes by fibres that are reported to exist in the normal heart (James, 196I).

The authors wish to acknowledge the co-operation of Dr. J. Shackleton, who gave permission to study this case; Dr. C. A. K. Bird, pathologist, Macclesfield Hospital, who kindly provided the specimen; Dr. K. Lubkiewicz, who performed the initial examination of the heart; Mrs. A. Smith, who performed the histological techniques, and Messrs. K. Walters and R. Wylie, who produced the photomicrographs and photography.

\section{References}

Anderson, R. H., and Latham, R. A. (1971). The cellular architecture of the human atrioventricular node, with a note on its morphology in the presence of a left superior vena cava. Fournal of Anatomy, 109, 443.

Anderson, R. H., and Taylor, I. M. (1972). Development of atrioventricular specialized tissue in human heart. British Heart fournal, 34, 1205.

Durrer, D., Schuilenburg, R. M., and Wellens, H. J. J. (1970). Pre-excitation revisited. American fournal of Cardiology, 25, 690 .
Ferrer, M. I. (1967). New concepts relating to the pre-excitation syndrome. Fournal of the American Medical Association, $201,1038$.

Ferrer, M. I. (1970). Pre-excitation syndrome: mechanisms and treatment. In Arrhythmias, Cardiovascular Clinics, Vol. 2, No. 2, p. I17. Ed. by L. Dreifus. F. A. Davis, Philadelphia.

Hoffman, B. F., and Cranefield, P. (1960). Electrophysiology of the Heart. McGraw Hill, New York.

James, T. N. (I96I). Morphology of the human atrioventricular node, with remarks pertinent to its electrophysiology. American Heart fournal, 62, 756.

Kent, A. F. S. (1893). Researches on the structure and function of the mammalian heart. Fournal of Physiology, 14, 233.

Lown, B., Ganong, W. F., and Levine, S. A. (1952). The syndrome of short $P-R$ interval, normal $Q R S$ complex, and paroxysmal rapid heart action. Circulation, 5, 693.

Mahaim, I., and Benatt, A. (1937). Nouvelles recherches sur les connexions supérieures de la branche gauche du faisceau de His-Tavara avec la cloison interventriculaire. Cardiologia, r, 6r.

Requests for'reprints to Dr. R. H. Anderson, Department of Anatomy and Embryology, Institute of Child Health, University of Liverpool, Alder Hey Children's Hospital, Eaton Road, Liverpool LI2 2 AP. 